\title{
Distinguishing the dominant species of pathogen in ethmoidal sinusitis by sequencing DNA dataset analysis
}

\author{
JUNYI ZHANG ${ }^{1,2 *}$, SHUAI HE ${ }^{1,3 *}$, YUNCHUAN LI ${ }^{1,3}$, MINGGANG LV ${ }^{1}$, \\ HONGZHENG WEI ${ }^{1,3}$, BIN QU $^{1}$, YANI ZHENG ${ }^{3}$ and CHUNHUA HU ${ }^{3}$
}

\begin{abstract}
${ }^{1}$ Department of Otolaryngology, Harbin First Hospital, Harbin, Heilongjiang 150070; ${ }^{2}$ Department of Otolaryngology, Daqing Oilfield General Hospital, Daqing, Heilongjiang 163001; ${ }^{3}$ Department of Otolaryngology Head and Neck Surgery, State Key Laboratory of Otolaryngology Head and Neck Surgery of Ministry of Education, Beijing Tongren Hospital,
\end{abstract} Capital Medical University, Beijing 100730, P.R. China

Received February 3, 2018; Accepted July 26, 2018

DOI: $10.3892 /$ etm.2018.6726

\begin{abstract}
Identifying the predominant microbial species in patients with ethmoidal sinusitis is conducive to its successful treatment. The aim of the present study was to determine the microbial composition and the predominant fungal and bacterial species in patients with ethmoidal sinusitis. A sample was obtained from 3 patients with ethmoidal sinusitis and from the ethmoid sinus of 2 healthy volunteers. Those samples were sequenced using an Illumina/Solexa sequencing platform for mapping to human, fungal, and bacterial genomes. Fungal and bacterial expressions in those samples were analyzed through bioinformatics and statistical methods. The sequencing data revealed that the dominant fungal strains in the ethmoidal sinusitis samples compared with the healthy controls (8_S33 and 10_S9) were Aspergillus oryzae and Aspergillus flavus, and the dominant bacterial strains were Haemophilus influenzae and Haemophilus parainfluenzae. Together, these findings indicate that the development of ethmoidal sinusitis is associated with the presence of fungi and bacteria, which may benefit the successful diagnosis and treatment for patients with ethmoidal sinusitis.
\end{abstract}

\section{Introduction}

Ethmoidal sinusitis is a common disease affecting the ethmoid sinus. Ethmoid sinus is located in the ethmoid nasal cavity above the labyrinth $(1,2)$. Because of the limitations of previous nasal examination methods and clinical application

Correspondence to: Dr Yunchuan Li, Department of Otolaryngology, Harbin First Hospital, 151 Diduan Street, Harbin, Heilongjiang 150070, P.R. China

E-mail: liyunchuan1964@163.com

*Contributed equally

Key words: fungi, bacteria, high throughput sequencing, ethmoidal sinusitis of antibiotics, ethmoidal sinusitis is often misdiagnosed, which increases the difficulty of attaining successful surgical treatment and drug therapy (3). In recent years, due to the development of cold light source nasal endoscopy, which overcomes the limitations of visual examination and the extensive use of computed tomography/magnetic resonance imaging in otolaryngology, a greater number of patients with ethmoidal sinusitis are successfully identified $(4,5)$. The acute inflammation of ethmoid sinus is not obvious, and the clinical symptoms of chronic inflammation are subtle $(1,3)$. Head or occipital blunt pain is an important symptom in patients with ethmoidal sinusitis, accompanied by insomnia, forgetfulness, depression, dizziness and other symptoms (6). More serious, ethmoidal sinusitis leads to weakening of the patient's sense of smell as a result of closing to the olfactory area (6-8). Therefore, the diagnosis and treatment of ethmoidal sinusitis has become the focus of clinical research.

The sinus mucosa connecting with nasal mucosa, weakening function of the epithelial cilia in nasal sinuses, abnormal anatomy of ethmoid sinus, allergic reactions and mucosal polyps may lead to stenosis or obstruction of the ethmoid sinus (9). Changes of microcirculation in nasal sinuses may be caused by disturbance of ventilation and drainage in nasal cavity and paranasal sinuses (7,9). Simultaneously, changes of microcirculation including an increase of secretion retention and humidity, decrease of oxygen content, increase of sugar content and changes in $\mathrm{pH}$ provide suitable conditions for the growth and reproduction of pathogenic microorganisms (10-12). When the patient's immune system is inhibited as a result of consumptive diseases, metabolic diseases or long-term use of antibiotics, and in glucocorticoid patients, pathogens may easily invade and multiply rapidly $(13,14)$. Several studies have reported, following a direct smear and culture of the pathogen, that the majority of pathogens present were Aspergillus, and a large number of bacteria are also detected $(8,11)$. However, the predominant fungal and bacterial species remain unknown.

High throughput sequencing with its high output and high resolution features not only provides a wealth of genetic information, but also greatly reduces the cost and time of sequencing (15). The Illumina/Solexa sequencing platform can 
be used to analyze millions of samples, which overcomes the poor accuracy of conventional analysis technology (16). In the present study, Illumina/Solexa sequencing platform was used for quantitative evaluation of individual fungal and bacterial species in ethmoidal sinusitis, in order to analyze the microbial species composition, which may benefits the diagnosis and treatment of ethmoidal sinusitis patients.

\section{Patients and methods}

Ethics statement. The present study was performed in full compliance with bioethical laws in China. The collection and use of patient data in the present study was approved by The Ethics Committee of Beijing Tongren Hospital (Beijing, China). Written informed consent was obtained from all research subjects. Personal information from the hospital database was applied for research purposes.

Sample. Between February 2015 and August 2016, 3 patients (1 male and 2 females) with confirmed ethmoidal sinusitis admitted to Department of Otolaryngology, Harbin First Hospital (Harbin, China) were included in the present study (the experiment al group). The age range of the experimental group was 42-63 years (Table I). Samples of the layer of white purulent secretions and the dark brown or brown sediment content were obtained from patients with ethmoidal sinusitis during endoscopic sinus surgery without opening the ethmoid sinus to remove the lesions. Between February 2015 and August 2016, 2 volunteers (1 male and 1 female), who had no history of allergic rhinitis, inflammatory sinus disease, nasal polyps, nasal septum deviation, or histopathologic examinations or radiographic analysis of the nasal cavity and paranasal sinuses, were included as the healthy control group. Samples of ethmoid sinus tissue were obtained from 2 healthy volunteers using the nasal endoscope. The age of this control group was between 51 and 60 years (Table I). Subsequently, five samples (one from each individual) were used for high throughput sequencing. The three samples from the experimental group were 1_S25, 3_S8 and 5_S17, and the two samples from the control group were 8_S33 and 10_S9.

DNA library construction and Solexa sequencing. A Fungal/Bacterial DNA Purification kit (catalog no. KA4361; AmyJet Scientific Co., Ltd., Wuhan, China) was used for purifying total DNA from samples according to the manufacturer's protocol. Based on the manufacturer's instructions, a TruSeq DNA Sample Preparation kit (v2; Illumina, Inc., San Diego, CA, USA) was used for synthesizing the paired-end libraries, with approximate mean insert lengths of $200 \mathrm{bp}$. Prior to cluster generation, the Agilent DNA1000 kit (Agilent, 5067-1504) was used for assaying library concentration following the manufacturer's protocol and size with a 2100 Bioanalyzer (Agilent, Palo Alto, CA). Libraries were sequenced as 100-mer X2 using a Hi-Seq 2000 sequencer equipped with a paired-end module (Illumina, Inc.).

Data analysis. A program was written by the authors of the current study with Perl 5.0 (Perl, Shanghai, China; http://www. perlchina.org/) to manage the mapping results and perform statistical analysis. All bases on the underside of each read and low sequences were removed using the aforementioned Perl program by cutting off the first low-quality base (quality, $<10$; assessed according to instructions from https:/www. ncbi.nlm.nih.gov/genome/microbes/) at a read length $<30 \mathrm{bp}$. The paired-end reads were removed when one read length was $<30 \mathrm{bp}$. Then, the remaining reads were mapped to the human genome. The high-quality reads were mapped to the human genome from NCBI (https://www.ncbi.nlm.nih. gov/genome/microbes/) using soap2 2.21 software (http:/soap. genomics.org.cn/). The paired reads mapping to the human genome were counted and filtered using soap2 software. Mapping to the bacterial genome and fungal genome was performed similarly to the human genome.

The bacterial genome was obtained from NCBI (https://www.ncbi.nlm.nih.gov/genome/?term=bacteria) using soap2 software. The fungal genome was obtained from NCBI (https://www.ncbi.nlm.nih.gov/genome/?term=Fungi) using soap2 software. Finally, fungal and bacterial expression analysis was performed as detailed in Fig. 1, and the predominant fungal and bacterial species were analyzed with soap2 software.

Statistical analysis. Data are presented as the mean \pm standard error of the mean. $\mathrm{P}<0.05$ was considered to indicate a statistically significant difference.

\section{Results}

Overview of microbial species in ethmoidal sinusitis samples. Illumina Hi-Seq 2000 was used to sequence three ethmoidal sinusitis samples and two healthy ethmoid sinus samples. Then, bioinformatics and statistical analysis were used to analyze the expressions of fungal and bacterial species (Fig. 1). Based on the short DNA read, the longest single length was 126 bp (Table II). A total of 168 fungi and 867 bacteria were detected in the samples (data not shown). The sequencing data in Table II demonstrates that the number of high quality reads was 14,778,016 in sample 5_S17, which was $99.05 \%$ of the total number of reads in sample 5_S17 and the highest number of reads was 7,460,156 (length cut-off, $\geq 30 \mathrm{bp}$ ). The high-quality reads of the experimental samples were higher than that of the healthy group. The greatest number of reads (7460156) was recorded in 5_S17, and the lowest number of reads was in 3_S8 $(6,070,482)$. Following mapping to the human genome, sample 1_S25 exhibited the lowest similarity $(1.767 \%$; Table III). Following mapping to the bacterial genome, sample 5_S17 exhibited the greatest similarity (85.874\%; Table III). Furthermore, the proportion of fungi was lowest in sample 1_S25 (0.027\%; Table III).

Bacteria in ethmoidal sinusitis samples. Following mapping to the bacterial genome, the similarity in the experimental group, from highest to lowest, were sample 5_S17 (85.874\%), sample 3_S8 (52.421\%), and sample 1_S25 (10.447\%; Table III). The bacterial similarity in healthy subjects was markedly lower than in patients. The bacterial similarity in sample 10_S9 (healthy) was the lowest $(0.016 \%$; Table III). Furthermore, it was observed that sample 1_S25 contained the highest number of bacterial species (867), but there were only 132 bacteria in healthy sample 8_S33 (data not shown). Further, the 6 
Table I. Sample information in the present study.

\begin{tabular}{lllcrr}
\hline Group & Sample no. & Sex & Age (years) & Sampling position & Date of surgery \\
\hline A & 1_S25 & Female & 42 & Anterior ethmoidal cell & Feb 15, 2015 \\
A & 3_S8 & Male & 63 & Posterior ethmoidal cell & Apr 4, 2016 \\
A & 5_S17 & Female & 56 & Anterior ethmoidal cell & Sep 2, 2015 \\
B & 8_S33 & Female & 51 & Anterior ethmoidal cell & Aug 21, 2016 \\
B & 10_S9 & Male & 60 & Posterior ethmoidal cell & Nov 27, 2015
\end{tabular}

Group A, the experimental group, confirmed ethmoidal sinusitis patients; group B, healthy controls.

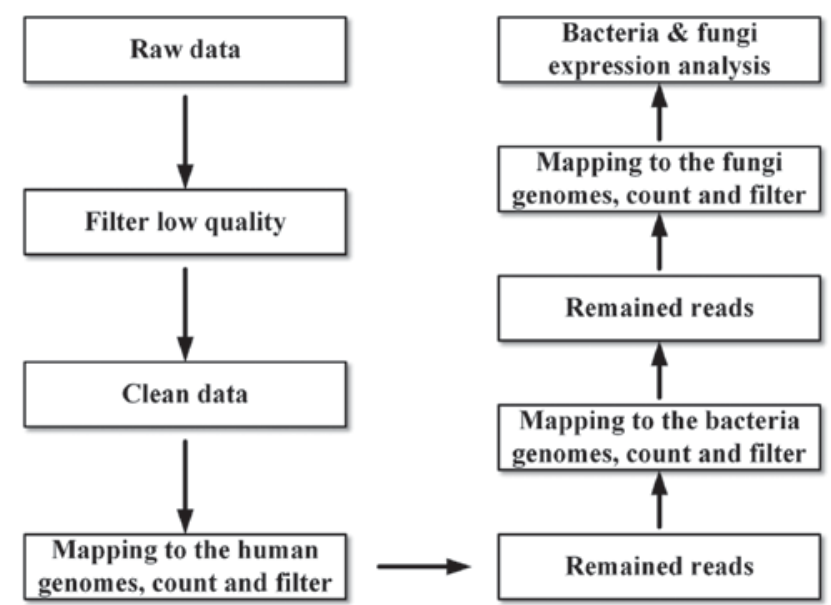

Figure 1. Flow diagram of the analytical procedure followed in the present study.

most frequent bacterial species were identified in the healthy and ethmoidal sinusitis samples. Enterobacter cloacae was the dominant bacterial species detected in 4 samples including 2 patients and 2 healthy controls (data not shown). Haemophilus influenzae and Haemophilus parainfluenzae were detected in 3 patient samples with similar levels of expression (Fig. 2). Klebsiella pneumoniae and Prevotella intermedia were detected in 3 samples with different levels of expression (Fig. 2). The most prevalent 6 bacterial species were also identified in the normal samples. Based on the analysis, it was identified that 4 of the most prevalent 6 strains in the normal samples were different from those of the patients. They were Haemophilus influenzae, Haemophilus parainfluenzae, Prevotella intermedia and Selenomonas sputigena (Fig. 2). This indicates notable differences in bacterial species between the healthy groups (8_S33 and 10_S9) and patients with ethmoidal sinusitis. Haemophilus influenzae and Haemophilus parainfluenzae were the dominant bacterial species in the ethmoidal sinusitis samples compared with the control.

Fungi in ethmoidal sinusitis samples. The remaining reads were mapped to the fungal genome. The analysis presented in Table III demonstrated that sample 8_S33 had the highest fungal composition $(0.320 \%)$ and 1_S25 had the lowest fungal composition $(0.027 \%)$. The similarity in the healthy group was markedly greater than that in the experimental group. Furthermore, there were 168 fungi in normal sample 10_S9, but only 73 fungi were detected in sample 1_S25 (experimental group; data not shown). The 6 most common fungi species in the experimental and healthy groups were also identified. Aspergillus rambellii was the most common fungi species detected in all 5 samples. The expression of Aspergillus rambellii in the experimental group was markedly lower compared with that in the healthy group $(\mathrm{P}<0.05)$. Pseudogymnoascus pannorum was detected in 4 samples. Mitosporidium daphniae was detected in 3 samples with different expressions. Aspergillus oryzae and Aspergillusflavus were detected in two samples of the experimental group. In 8_S33 (33142) Mitosporidium daphniae was detected with the highest level, followed by Aspergillus oryzae in sample 5_S17. Aspergillus oryzae and Aspergillus flavus were the dominant fungal species observed in patient samples, while absent in the control (Fig. 3).

Other microbia identified in subject samples. Following mapping to human, bacterial and fungal genomes, sample 1_S25 exhibited the highest prevalence of unknown reads (87.758\%, experimental group), and the lowest prevalence of unknown reads was in sample 5_S17 in the experiment group (9.633\%). Sample 8_S33 and sample 10_S9 in the healthy group had similar prevalences of unknown reads (Table III).

\section{Discussion}

Ethmoidal sinusitis occurs in the unilateral sinus, and can be identified via the thickening of the ethmoid sinus mucosa, bone destruction at the top of the sieve, polyps obstruction in the meatus nasi medius, olfactory cleft and purulent secretion in the middle meatus in the meatus nasi medius $(17,18)$. Ethmoidal sinusitis is usually classified as acute ethmoidal sinusitis and chronic ethmoidal sinusitis $(3,7)$. Acute ethmoidal sinusitis exhibits an acute onset and rapid progress caused by acute rhinitis (2). Chronic ethmoidal sinusitis is caused by acute ethmoidal sinusitis that cannot be completely cured or recurrent acute ethmoidal sinusitis (19). Chronic sinusitis is often associated with chronic maxillary sinusitis (14). Pathogenic microorganisms penetrate the mucosal barrier to invade blood vessels or bone, which can cause vasculitis, vascular embolization, bone destruction and tissue necrosis (17). Severe infections of the ethmoid sinus often lead to fungal meningitis, encephalitis and brain necrosis, which, altogether, have a mortality rate between 50 and $100 \%$ (20). Therefore, 
Table II. Analysis of the reads identified in sequencing samples.

\begin{tabular}{lcccccc}
\hline & & & & & \multicolumn{2}{c}{ High quality } \\
\cline { 5 - 7 } Sample & $\begin{array}{c}\text { Short reads } \\
(\mathrm{n})\end{array}$ & $\begin{array}{c}\text { Single length } \\
(\mathrm{bp})\end{array}$ & $\begin{array}{c}\text { Total length } \\
(\mathrm{bp})\end{array}$ & $\begin{array}{c}\text { Reads } \\
(\mathrm{n})\end{array}$ & $\begin{array}{c}\text { Total length } \\
\text { (bp) }\end{array}$ & $\begin{array}{c}\text { High quality read out } \\
\text { of total reads }(\%)\end{array}$ \\
\hline 1_S25 & $6,651,991$ & $35-126$ & $1,665,728,087$ & $13,062,670$ & $1,612,535,682$ & 98.19 \\
3_S8 & $6,070,482$ & $35-126$ & $1,516,014,942$ & $11,963,776$ & $1,475,872,767$ & 98.54 \\
5_S17 & $7,460,156$ & $35-126$ & $1,865,910,724$ & $14,778,016$ & $1,831,326,470$ & 99.05 \\
8_S33 & $6,721,553$ & $35-126$ & $1,677,084,576$ & $13,095,690$ & $1,612,590,049$ & 97.42 \\
10_S9 & $6,497,648$ & $35-126$ & $1,619,862,741$ & $12,663,024$ & $1,556,099,543$ & 97.44 \\
\hline
\end{tabular}

With a experimental group and ${ }^{\mathrm{b}}$ healthy controls; length cut-off, $30 \mathrm{bp}$.

Table III. Reads species composition in sequencing samples.

\begin{tabular}{|c|c|c|c|c|c|c|c|c|c|c|}
\hline \multirow[b]{2}{*}{ Sample } & \multicolumn{2}{|c|}{$\begin{array}{c}\text { Total high quality } \\
\text { reads }\end{array}$} & \multicolumn{2}{|c|}{ Homo sapiens } & \multicolumn{2}{|c|}{ Bacterial } & \multicolumn{2}{|c|}{ Fungal } & \multicolumn{2}{|c|}{ Unknown microbes } \\
\hline & $\mathrm{n}$ & $\%$ & $\mathrm{n}$ & $\%$ & $\mathrm{n}$ & $\%$ & $\mathrm{n}$ & $\%$ & $\mathrm{n}$ & $\%$ \\
\hline 1_S25 & $13,062,670$ & 100 & 230,863 & 1.767 & $1,364,683$ & 10.447 & 3,591 & 0.027 & $11,463,533$ & 87.758 \\
\hline 3_S8 ${ }^{\mathrm{a}}$ & $11,963,776$ & 100 & $2,316,555$ & 19.363 & $6,271,526$ & 52.421 & 16,857 & 0.141 & $3,358,838$ & 28.075 \\
\hline 5_S17 ${ }^{\mathrm{a}}$ & $14,778,016$ & 100 & 629,593 & 4.260 & $12,690,426$ & 85.874 & 34,372 & 0.233 & $1,423,625$ & 9.633 \\
\hline 8_S33 ${ }^{\mathrm{b}}$ & $13,095,690$ & 100 & $11,407,813$ & 87.111 & 96,861 & 0.740 & 41,890 & 0.320 & $1,549,126$ & 11.829 \\
\hline 10_S9b & $12,663,024$ & 100 & $11,212,700$ & 88.547 & 2,028 & 0.016 & 36,193 & 0.286 & $1,412,103$ & 11.151 \\
\hline
\end{tabular}

With aexperimental group and bealthy controls. Soap2 2.21 software was used for this analysis. Homo sapiens, default parameters; bacterial and fungal parameters, -130 .

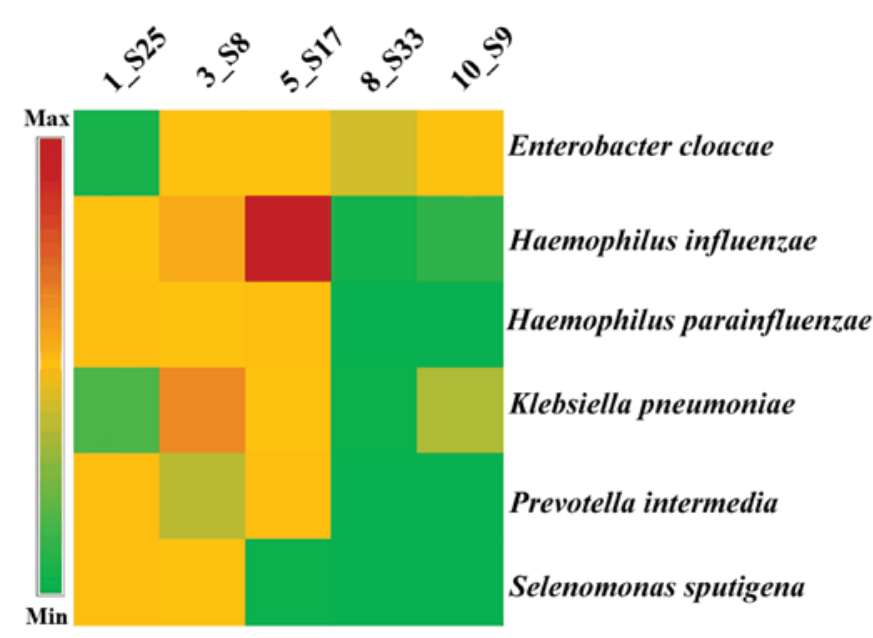

Figure 2. One-way hierarchical cluster map of the top six bacterial species.

it is of importance to the diagnosis and treatment of patients to elucidate the microbial composition and the predominant species of ethmoidal sinusitis. In the current study, all cases were treated via endoscopic sinus surgery without opening the ethmoid sinus to remove the lesions. The ethmoidal sinusitis samples and ethmoid sinus samples were analyzed using an Illumina/Solexa sequencing platform. Subsequently, the reads were mapped to human, fungal, and bacterial genomes, and

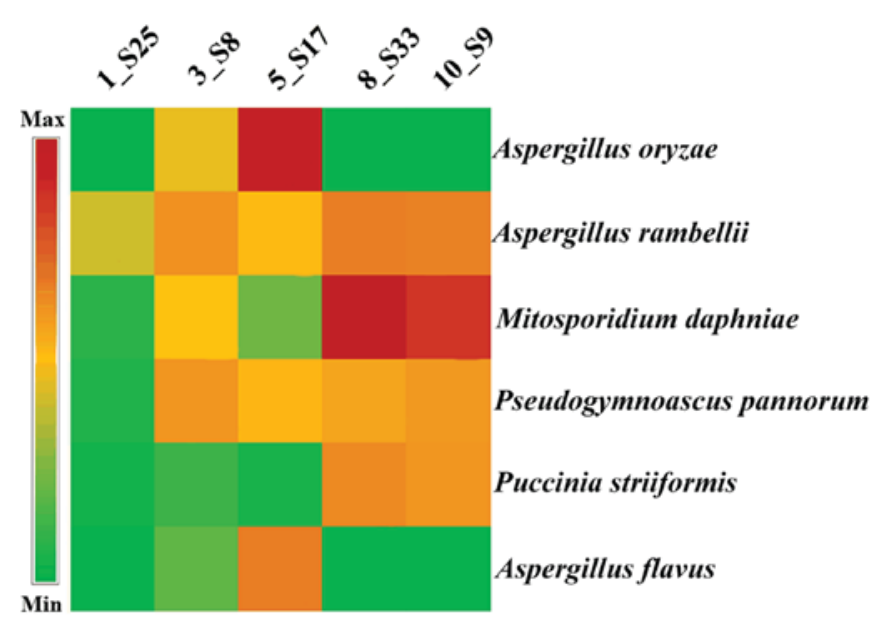

Figure 3. One-way hierarchical cluster map of the top six fungal species.

the predominant fungal and bacterial species were globally analyzed to identify the microbial composition in those samples.

With the rapid development of sequencing technology, high throughput sequencing technology has been used increasingly to solve biological problems. For example, the reference sequences of species were not obtained through de novo sequencing, which lays the foundation to further 
research molecular breeding; genome sequencing is carried out for species with reference sequences to research the molecular basis of the individual differences (21-23). The Illumina/Solexa sequencing platform is one high throughput sequencing method that allows sequencing by synthesis, which is built on the principle of proprietary reversible chemical reactions (24). Sequencing analysis of hundreds of thousands of DNA molecules makes it possible to obtain a detailed picture of the transcriptome and genome of a species $(25,26)$. High throughput sequencing technology includes high output and high-resolution features that provides a wealth of genetic information, and also greatly reduces the cost and time of sequencing (27). Therefore, the Illumina/Solexa sequencing platform may facilitate the rapid and accurate detection of the microbial composition and the predominant fungal and bacterial species in patients with ethmoidal sinusitis.

The majority of the fungal infections in the ethmoid sinus are non-invasive fungal balls (25). It has previously been demonstrated that the fungal pathogens of fungal ethmoidal sinusitis were mainly Aspergillus (28). In the present study, it was observed that Aspergillus oryzae and Aspergillus flavus were the dominant fungal strains in ethmoidal sinusitis compared with the healthy controls by high throughput sequencing, as the expression of those species were markedly higher compared with the healthy group. Fungi are widely found in nature, and fungal spores that often mix with dust and air are easily absorbed into the human respiratory tract (29). Risk factors, including the weakness of mucosa epithelial cilia function under sinus, anatomical abnormality of recessus sphenoethmoidalis, allergy and mucosal polyp cause poor nasal drainage. Additionally, the immune system is dysfunctional, thus fungal hyphae or spores will reproduce in the layer of mucous membrane and mucous membrane of the nasal cavity, which communicates with the nasal cavity $(30,31)$. A warm and moist environment in the ethmoid sinus induces fungal growth and reproduction, which gradually forms a colony. The colony is likely to block the natural opening of the sinuses and cause infection (32).

Aspergillus is an opportunistic pathogen; healthy people have a strong resistance to Aspergillus, but the infection risk is increased when the patients exhibit the following pathological conditions: i) The patients are immunosuppressed, ii) the patients are in a vulnerable state, for example they are diagnosed with a terminal disease, iii) the patients have undergone prolonged use of antibiotics that induce dysbacteriosis, iv) the patients exhibiting weakened local resistance at trauma sites, v) the patients are in an Aspergillus-rich environment for a long time (33-35). The present findings indicated that Aspergillus oryzae and Aspergillus flavus are associated with ethmoidal sinusitis. It has also been reported that ethmoidal sinusitis is caused by fungal or mixed fungal and bacterial infections (20). It was further identified that Haemophilus influenzae and Haemophilus parainfluenzae were the dominant bacterial species in the ethmoidal sinusitis samples, whereas Aspergillus rambellii, Mitosporidium daphniae, Pseudogymnoascus pannorum and Puccinia striiformis were the dominant fungal species in the healthy samples. There were notable differences in bacterial species between the healthy and experimental groups. Secretion retention, moisture and sugar content increases in the ethmoid sinus create suitable conditions for the growth and reproduction of bacteria. Bacteria then invade the mucosal epithelium, which attracts inflammatory cells and promotes the release of prostaglandin and histamine through the flagella and capsule, and the release of collagenase and protease (36). This leads to the development of mucosal injury and inflammation. It was also identified that the similarity of bacteria in the experimental groups was significantly higher than that in the healthy group, whereas the similarity of fungi in the experimental groups was markedly lower than that in the healthy group. The possible reason is that $P$. aeruginosa restrained fungal growth, which weakened the fungal hyphae fractured (37). These findings suggest that fungal and bacterial presence is associated with the development of ethmoidal sinusitis.

Following sequencing the ethmoidal sinusitis samples, the microbial composition and the predominant species of fungal and bacterial was analyzed globally in the samples. The sequencing data indicated that compared to the healthy control the dominant fungal strains in ethmoidal sinusitis samples were Aspergillus oryzae and Aspergillus flavus, and the dominant bacterial strains were Haemophilus influenzae and Haemophilus parainfluenzae. These findings suggest that the development of fungal ethmoidal sinusitis is associated with the fungi and bacteria. However, there are still certain limitations in to the current study, such as the small sample size. In the future, the authors of the current study would like to expand the sample size so a more credible conclusion may be reached.

\section{Acknowledgements}

Not applicable.

\section{Funding}

ThepresentstudywassupportedbyTalentResearchProjectsintroduced by Harbin First Hospital (grant no. 2014SYYRCYJ05) and a grant from Wu Jieping Clinical Research Special Assistance Fund (grant no. 320.6750.15066).

\section{Availability of data and materials}

All data generated or analyzed during the present study are included in this published article.

\section{Authors' contributions}

JZ, SH and YL were responsible for study design and planning. ML was responsible for data collection and entry. HW was responsible for data analysis and statistics. YZ was responsible for data interpretation. JZ and SH were responsible for manuscript preparation. BQ and $\mathrm{CH}$ were responsible for literature analysis and search. YL was responsible for funds collection. All authors read and approved the final manuscript.

\section{Ethics approval and consent to participate}

The present study was approved by the Ethics Committee of Beijing Tongren Hospital (Beijing, China). Written informed consent was obtained from all patients and/or their guardians. 


\section{Patient consent for publication}

Patients or their guardians provided written informed consents for publication.

\section{Competing interests}

The authors declare that they have no competing interests.

\section{References}

1. McArdle B and Perry C: Ethmoid silent sinus syndrome causing inward displacement of the orbit: Case report. J Laryngol Otol 124: 206-208, 2010

2. Yue JX, Zhang S, Kong WJ, Wang YJ, Xiong XG and Zhu LX: Trans-superior meatus endoscopic surgery of sphenoidal sinus and sellar area: A surgical technique for lesion of sellar area Acta Otolaryngol 128: 1233-1237, 2008.

3. Mayr SI, Hafizovic K, Waldfahrer F, Iro H and Kütting B: Characterization of initial clinical symptoms and risk factors for sinonasal adenocarcinomas: Results of a case-control study. Int Arch Occup Environ Health 83: 631-638, 2010.

4. Hoang JK, Eastwood JD, Tebbit CL and Glastonbury CM: Multiplanar sinus CT: A systematic approach to imaging before functional endoscopic sinus surgery. AJR Am J Roentgenol 194: W527-W536, 2010.

5. Park IH, Song JS, Choi H, Kim TH, Hoon S, Lee SH and Lee HM: Volumetric study in the development of paranasal sinuses by CT imaging in Asian: A pilot study. Int J Pediatr Otorhinolaryngol 74: 1347-1350, 2010.

6. Velasquez N, Thamboo A, Abuzeid WM and Nayak JV: Safe treatment of ethmoid sinusitis utilizing minimally invasive ethmoid punch sinusotomy in chronic rhinosinusitis without polyposis patients. Laryngoscope 127: 1268-1275, 2017.

7. Rombaux P, Gigi J, Hamoir M, Eloy P and Bertrand B: Bacteriology of chronic sinusitis: The bulla ethmoidalis content. Rhinology 40: 18-23, 2002.

8. Togitani K, Kobayashi M, Sakai M, Uemura Y, Taguchi H, Morita T, Sugihara S, Sano A and Nishimura K: Ethmoidal sinusitis caused by Exserohilum rostratum in a patient with malignant lymphoma after non-myeloablative allogeneic peripheral blood stem cell transplantation. Transpl Infect Dis 9: 137-141, 2007.

9. Pradhan P, Gochhait D and Kumar A: Squamous cell carcinoma of cervix with isolated metastasis to ethmoidal sinus: A rare occurrence. J Clin Diagn Res 11: ED01-ED02, 2017.

10. Zang Y, Wei Y, Shi Y, Chen Q and Xing D: Chemo/photoacoustic dual therapy with mRNA-triggered DOX release and photoinduced shockwave based on a DNA-Gold nanoplatform. Small 12: 756-769, 2016

11. Park EH, Mahanty B, Yoon SU and Kim CG: Biomimetic acid soil amelioration using indigenous soil isolates in comparison to other potential microorganism. Commun Soil Sci Plant Anal 47: 1296-1305, 2016.

12. Haug RH: Microorganisms of the nose and paranasal sinuses. Oral Maxillofac Surg Clin North Am 24: 191-196, 2012.

13. Yan L, Hu F, Yan X, Wei Y, Ma W, Wang Y, Lu S and Wang Z: Inhibition of microRNA-155 ameliorates experimental autoimmune myocarditis by modulating Th17/Treg immune response. J Mol Med (Berl) 94: 1063-1079, 2016.

14. Alten R and Wiebe E: Hypothalamic-pituitary-adrenal axis function in patients with rheumatoid arthritis treated with different glucocorticoid approaches. Neuroimmunomodulat 22: 83-88, 2015.

15. Massana R, Gobet A, Audic S, Bass D, Bittner L, Boutte C, Chambouvet A, Christen R, Claverie JM, Decelle J, et al: Marine protist diversity in European coastal waters and sediments as revealed by high-throughput sequencing. Environ Microbiol 17: 4035-4049, 2015.

16. Hu Z, Cheng L and Wang H: The Illumina-solexa sequencing protocol for bacterial genomes. Methods Mol Biol 1231: 91-97, 2015.

17. Crovetto-Martinez R, Martin-Arregui FJ, Zabala-Lópezde-Maturana A, Tudela-Cabello K and Crovetto-de la Torre MA Frequency of the odontogenic maxillary sinusitis extended to the anterior ethmoid sinus and response to surgical treatment. Med Oral Patol Oral Cir Bucal 19: e409-e413, 2014.
18. Sanz-Marco E, Espana E, Alamar A, Perez-Rojas J, Lopez-Prats MJ and Diaz-Llopis M: Orbital alveolar rhabdomyosarcoma masked by ethmoid sinusitis in a 25-year-old. Arch Soc Esp Oftalmol 89: 182-185, 2014 (In Spanish).

19. Bergeron M and Leclerc JE: Is image guidance accurate in children sinus surgery? Int J Pediatr Otorhinolaryngol 79: 469-473, 2015.

20. Sorichetti B, Westerberg BD, Tan R and Kozak FK: Nocardia asteroides sinusitis in a pediatric patient: Case report with 20 year follow-up and review of the literature. Int J Pediatr Otorhinolaryngol 79: 1152-1154, 2015.

21. Goodwin S, McPherson JD and McCombie WR: Coming of age: Ten years of next-generation sequencing technologies. Nat Rev Genet 17: 333-351, 2016.

22. Baker MW, Atkins AE, Cordovado SK, Hendrix M, Earley MC and Farrell PM: Improving newborn screening for cystic fibrosis using next-generation sequencing technology: A technical feasibility study. Genet Med 18: 231-238, 2016.

23. Hosomichi K, Shiina T, Tajima A and Inoue I: The impact of next-generation sequencing technologies on HLA research. J Hum Genet 60: 665-673, 2015.

24. Yi S, Gao ZX, Zhao H, Zeng C, Luo W, Chen B and Wang WM: Identification and characterization of microRNAs involved in growth of blunt snout bream (Megalobrama amblycephala) by Solexa sequencing. BMC Genomics 14: 754, 2013.

25. Fan XD, Wang JQ, Yang N, Dong YY, Liu L, Wang FW, Wang N, Chen H, Liu WC, Sun YP, et al: Gene expression profiling of soybean leaves and roots under salt, saline-alkali and drought stress by high-throughput Illumina sequencing. Gene 512: 392-402, 2013

26. Qi P, Guo B, Zhu A, Wu C and Liu C: Identification and comparative analysis of the Pseudosciaena crocea microRNA transcriptome response to poly(I:C) infection using a deep sequencing approach. Fish Shellfish Immunol 39: 483-491, 2014.

27. Morgan S, Shoai M, Fratta P, Sidle K, Orrell R, Sweeney MG, Shatunov A, Sproviero W, Jones A, Al-Chalabi A, et al: Investigation of next-generation sequencing technologies as a diagnostic tool for amyotrophic lateral sclerosis. Neurobiol Aging 36: 1600.e5-e8, 2015.

28. Zhang J, Li Y, Lu X, Wang X, Zang H, Wang T, Zhou B and Zhang L: Distinguishing the dominant species of pathogen in maxillary sinusitis by sequencing DNA dataset analysis. Gene 561: 256-260, 2015.

29. Koljalg U, Nilsson RH, Abarenkov K, Tedersoo L, Taylor AF, Bahram M, Bates ST, Bruns TD, Bengtsson-Palme J, Callaghan TM, et al: Towards a unified paradigm for sequence-based identification of fungi. Mol Ecol 22: 5271-5277, 2013.

30. Yan J and Li Y: Extracranial orbital meningioma extending into the nasosinus in neurofibromatosis type 2. J Craniofac Surg 25: 664-666, 2014.

31. Zainine R, Loukil I, Dhaouadi A, Ennaili M, Mediouni A, Chahed H, Beltaief N and Besbes G: Ophthalmic complications of nasosinus mucoceles. J Fr Ophtalmol 37: 93-98, 2014 (In French).

32. Kitamura T, Takemoto H, Makinae H, Yamaguchi T and Maki K: A preliminary acoustic analysis of three-dimensional shape of the human nasal cavity and paranasal sinuses extracted from cone-beam CT. J Acoust Soc Am 140: 3223, 2016.

33. Hicks JK, Gonzalez BE, Zembillas AS, Kusick K, Murthy S, Raja S, Gordon SM and Hanna R: Invasive aspergillus infection requiring lobectomy in a CYP2C19 rapid metabolizer with subtherapeutic voriconazole concentrations. Pharmacogenomics 17: 663-667, 2016.

34. Peghin M, Monforte V, Martin-Gomez MT, Ruiz-Camps I, Berastegui C, Saez B, Riera J, Ussetti P, Solé J, Gavaldá J and Roman A: 10 years of prophylaxis with nebulized liposomal amphotericin B and the changing epidemiology of Aspergillus spp. Infection in lung transplantation. Transpl Int 29: 51-62, 2016.

35. Beitzen-Heineke A, Bouzani M, Schmitt AL, Kurzai O, Hünniger K, Einsele H and Loeffler J: Human invariant natural killer $\mathrm{T}$ cells possess immune-modulating functions during Aspergillus infection. Med Mycol 54: 169-176, 2016.

36. Pluhacek T, Petrik M, Luptakova D, Benada O, Palyzova A, Lemr K and Havlicek V: Aspergillus infection monitored by multimodal imaging in a rat model. Proteomics 16: 1785-1792, 2016.

37. Kerr J: Suppression of fungal growth exhibited by Pseudomonas aeruginosa. J Clin Microbiol 32: 525-527, 1994. 